\title{
Antitumor activity of gambogic acid on NCI-H1993 xenografts via MET signaling pathway downregulation
}

\author{
DONGLEI LI, HUIWEI YANG, RUNPU LI, YANLI WANG, WEIJUN WANG, \\ DONGJIE LI, SHAOLIN MA and XUYU ZHANG \\ Oncology Department, Baoding Second Central Hospital of Oncology, Zhuozhou, Hebei 072750, P.R. China
}

Received August 9, 2014; Accepted April 24, 2015

DOI: $10.3892 / \mathrm{ol} .2015 .3719$

\begin{abstract}
The present study aimed to investigate the anti-tumor mechanisms of gambogic acid (GA) on NCI-H1993 xenografts in vivo. Non-small cell lung carcinoma NCI-H1993 cells, which harbor a $M E T$ gene amplification, were subcutaneously injected into athymic nude mice. The mice were randomly assigned to treatment with 10,20 or $30 \mathrm{mg} / \mathrm{kg}$ GA for 3 weeks. At the end of the efficacy study, all the mice were sacrificed and the tumor tissues were subjected to western blot analysis and immunohistochemical (IHC) staining. GA inhibited NCI-H1993 xenograft tumor growth in a dose-dependent manner. Western blot analysis demonstrated that expression of phosphorylated (p)-MET and its downstream signaling molecules p-AKT and p-ERK1/2 were significantly inhibited by GA. IHC analysis of $\mathrm{Ki}-67$ expression demonstrated that GA treatment resulted in dose-dependent inhibition of tumor cell proliferation. GA exerted antitumor effects on NCI-H1993 xenografts in vivo by direct regulation of the MET signaling pathway. Theses antitumor effects were primarily a result of its anti-proliferation function.
\end{abstract}

\section{Introduction}

The proto-oncogene $M E T$ encodes the receptor tyrosine kinase MET. Its primary function is to mediate morphogenetic embryonic development and tissue repair in vertebrates. The natural ligand for this receptor is the hepatocyte growth factor; the binding of this ligand to MET induces tyrosine phosphorylation of the receptor and activation of downstream signaling pathways mediated by phosphoinositide 3-kinase and AKT, by signal transducer and activator of transcription 3, or RAS and mitogen-activated protein kinase (MAPK) $(1,2)$.

Correspondence to: Dr Donglei Li, Oncology Department, Baoding Second Central Hospital of Oncology, 57 Fanyangzhong Road, Zhuozhou, Hebei 072750, P.R. China

E-mail: dongleili1980@126.com

Key words: gambogic acid, MET, non-small cell lung cancer, NCI-H1993, receptor tyrosine kinase
Garcinia hanburyi (G. hanburyi) is a traditional herbal medicine, which is used for anti-inflammation and hemostasis in South Asia. Gambogic acid (GA) is the main active component extracted from G. hanburyi. Gamboge resin has previously been used as a coloring material and in Traditional Chinese Medicine for the treatment of human diseases (3). A previous study demonstrated that GA exerts antitumor effects in vitro and in vivo (4). GA has been demonstrated to inhibit proliferation, induce apoptosis, reverse multidrug resistance and possess anti-angiogenic properties (5).

GA has been approved by the Chinese Food and Drug Administration for the treatment of different types of cancer in clinical trials $(6,7)$. Therefore, identification of the specific molecular targets responsible for the observed GA-mediated antitumor effects may be of clinical significance. A number of potential molecular targets of GA have been reported, which may contribute to its cytotoxic and antitumor activities, including binding to the transferrin receptor, suppressing nuclear factor $-\kappa \mathrm{B}(\mathrm{NF}-\kappa \mathrm{B})$ signaling transduction (8) and inhibiting the KDR signaling pathway (9). GA was also found to induce apoptosis in the non-small cell lung cancer (NSCLC) cell lines SPC-A1 and SK-MES-1 via Caspase 2, Caspase 9, Caspase 10, Bax and involved signaling pathways (10). Lung cancer is the leading cause of cancer mortalities worldwide, accounting for $18.2 \%$ of all cancers. The ratio of mortality to incidence is 0.86 , and NSCLC represents $\sim 80 \%$ of all lung cancers (11).

Although GA has been demonstrated to exert an antitumor effect on NSCLC, there are few reports regarding the mechanisms underlying this activity at present. The current study aimed to elucidate the potential mechanisms involved.

\section{Materials and methods}

Reagents. GA was purchased from Sigma-Aldrich (St. Louis, MO, USA). The MET selective inhibitor, PHA665752, was purchased from Selleck Chemicals (Houston, TX, USA). All drugs used in the present study were dissolved in sterile dimethylsulfoxide (DMSO; Sigma-Aldrich); a $10 \mathrm{mM}$ working solution was prepared and stored in aliquots at $-22^{\circ} \mathrm{C}$. Rabbit polyclonal $\operatorname{IgG}$ antibodies against human phosphorylated (p-) MET (\#sc-101736), p-AKT (\#sc-101629), p-extracellular-signal-regulated kinase (ERK; \#sc-101760), MET (\#sc-10), AKT (\#sc-8312) and ERK (\#sc-292838) were purchased from Santa Cruz Biotechnology, Inc. (Dallas, TX, 
USA). Rabbit anti-Ki-67 monoclonal IgG antibodies for immunohistochemistry (IHC) were purchased from Cell Signaling Technology, Inc. (Danvers, MA, USA; \#9129). All the chemicals used in the present study were of analytical reagent grade.

Cell culture. The human NSCLC cell line, NCI-H1993, which harbors a MET gene amplification (12), was obtained from the American Type Culture Collection (Manassas, VA, USA). The cells were cultured in DMEM supplemented with $10 \%$ fetal bovine serum, $10^{5} \mathrm{U} / 1$ penicillin and $100 \mathrm{mg} / 1$ streptomycin (GE Healthcare Life Sciences , Logan, UT, USA) at $37^{\circ} \mathrm{C}$ in an atmosphere containing $5 \% \mathrm{CO}_{2}$.

Animals. BALB/c female nude mice, obtained from Vital River Laboratory Animal Technology Co., Ltd. (Beijing, China), were used when they were 7-9 weeks old. The health of all animals was monitored daily by gross observation, and the experimental animals were housed in the laminar airflow cabinet. All the animals were allowed to acclimatize and recover from any stress associated with shipping for at least three days prior to experimental manipulation. Autoclaved water and irradiated food (Vital River Laboratory Animal Technology Co., Ltd.) were provided ad libitum, and the animals were maintained in a $12 \mathrm{~h}$ light and dark cycle. Cages, bedding and water bottles were autoclaved prior to use and were changed twice weekly. All the animal experiments were performed in accordance with protocols approved by the Experimental Animal Center of the Second Military Medical University Animal Care and Use Committee (Zhuozhou, China).

NCI-H1993 xenograft model establishment. NCI-H1993 cells were harvested, pelleted by centrifugation at $600 \mathrm{x} g$ for $10 \mathrm{~min}$, and resuspended in sterile serum-free medium supplemented with 50\% Matrigel (BD Biosciences, Franklin Lakes, NJ, USA). The cells $\left(5 \times 10^{6}\right.$ in $\left.100 \mu \mathrm{l}\right)$ were then subcutaneously implanted into the hind-flank region of each mouse and allowed to grow to a volume of $150-200 \mathrm{~mm}^{3}$ prior to the administration of GA.

Efficacy study. Nude mice bearing NCI-H1993 tumors $\left(150-200 \mathrm{~mm}^{3}\right)$ received vehicle [10\% DMSO, 15\% ethanol and $75 \%$ phosphate-buffered saline (PBS) (Sigma-Aldrich)] or 10,20 or $30 \mathrm{mg} / \mathrm{kg}$ GA (10\% DMSO, $15 \%$ ethanol and $75 \%$ PBS via intraperitoneal (i.p.) injection; or $10 \mathrm{mg} / \mathrm{kg}$ PHA665752 (L-lactate and 10\% polyethylene glycol; Selleck Chemicals, Houston, TX, USA) via tail intravenous (i.v.) injection for 21 consecutive days. On day 21 of the efficacy study, at $2 \mathrm{~h}$ following the final treatment with GA, the mice were humanely sacrificed by $\mathrm{CO}_{2}$ overexposure, and the tumors were resected. The tumor volume (TV) was determined by measurement with electronic vernier calipers, and the TV was calculated using the formula: $\mathrm{TV}=$ length $\mathrm{x}$ width ${ }^{2} / 2$. TV was expressed on the indicated days as the median $\mathrm{TV} \pm$ standard deviation for the indicated groups of mice.

Western blotting analysis. The protein expression levels of p-MET, p-AKT, p-ERK, MET, AKT and ERK were analyzed by western blot analysis. On day 21 of the efficacy study, at $2 \mathrm{~h}$ following the final injection of GA, the mice were humanely sacrificed. The tumors were harvested in lysis buffer (Cell Signaling Technology, Inc.) and homogenized using Misonix
Sonicator 4000 (Misonix Inc., Farmingdale, NY, USA); protein lysates were generated and protein concentrations were determined using a bicinchoninic acid assay (Pierce Biotechnology, Inc., Rockford, IL, USA). Equal amounts of protein (50 $\mu \mathrm{g})$ were then separated by SDS-PAGE on $10 \%$ gels, blotted on polyvinylidene difluoride membranes (Sigma-Aldrich) and probed with p-MET, p-AKT, p-ERK, MET, AKT and ERK rabbit polyclonal primary antibodies (dilution, 1:1,000; incubation, overnight at $4^{\circ} \mathrm{C}$ ) and subsequently with goat anti-rabbit horseradish peroxidase-conjugated secondary antibody (\#sc-2040; dilution, 1:1,000; incubation, $1 \mathrm{~h}$ at room temperature), and detected with an enhanced chemiluminescence kit (Sigma-Aldrich).

IHC. The tumor specimens were fixed in $10 \%$ buffered formalin for $24 \mathrm{~h}$ prior to being transferred to $70 \%$ ethanol. The tumor samples were subsequently paraffin-embedded, and 4-mm sections were cut and baked onto microscope slides (formalin, paraffin and slides from Sigma-Aldrich). The slides were incubated with the primary Ki-67 antibody (dilution, 1:1,000; incubation, overnight at $4^{\circ} \mathrm{C}$ ), then secondary antibodies (dilution, 1:1,000; incubation, $1 \mathrm{~h}$ at room temperature), and visualized using a colorimetric method (EnVision+ System-HRP DAB kit; Dako North America, Inc., Carpinteria, CA, USA). All of the immunostained sections were counterstained using hematoxylin. An automated Ventana Discovery XT Staining Module (Ventana Medical Systems, Inc., Tucson, AZ, USA) was used to conduct histological staining. The stained sections were analyzed using an Olympus BX46 microscope (Olympus Corporation, Tokyo, Japan), and quantitative analysis of section staining was performed using the Automated Cellular Imaging system (GE Healthcare Life Sciences, Chalfont, UK). The number of Ki-67-positive nuclei was counted regardless of the immunointensity in 4 random fields at x100 magnification (60\% center field).

Caspase activity assay. As a measure of the level of apoptosis, caspase-3, -8 and -9 activity were measured using caspase colorimetric protease kits (Abnova Corporation, Walnut, CA, USA). Fresh tumors in each group were resected following the final treatment with 10,20 or $30 \mathrm{mg} / \mathrm{kg}$ GA for $2 \mathrm{~h}$ on day 21 of the efficacy study, and the tumor lysis containing $200 \mu \mathrm{g}$ protein was incubated with $5 \mu \mathrm{l} 4 \mathrm{mM}$ pNA-conjugated caspase substrates (4-amino acid sequences; DEVD-pNA, IETD-pNA and LEHD-pNA) at $37^{\circ} \mathrm{C}$ for $2 \mathrm{~h}$. The quantity of pNA released was measured at $405 \mathrm{~nm}$ using a FLx $800^{\mathrm{TM}}$ Multi-Detection microplate reader (BioTek Instruments, Inc., Winooski, VT, USA).

Statistical analysis. All the results and data were confirmed in at least three separate experiments. The data are expressed as the mean \pm standard deviation, and were analyzed by Student's t-test using SPSS software, version 13.0 (SPSS Inc., Chicago, IL, USA). $\mathrm{P}<0.05$ was considered to indicate a statistically significant difference.

\section{Results}

GA inhibits tumor growth of NCI-H1993 xenografts. In order to investigate the tumor growth inhibition effect of GA on NCI-H1993 xenografts in vivo, NCI-H1993 tumor-bearing 


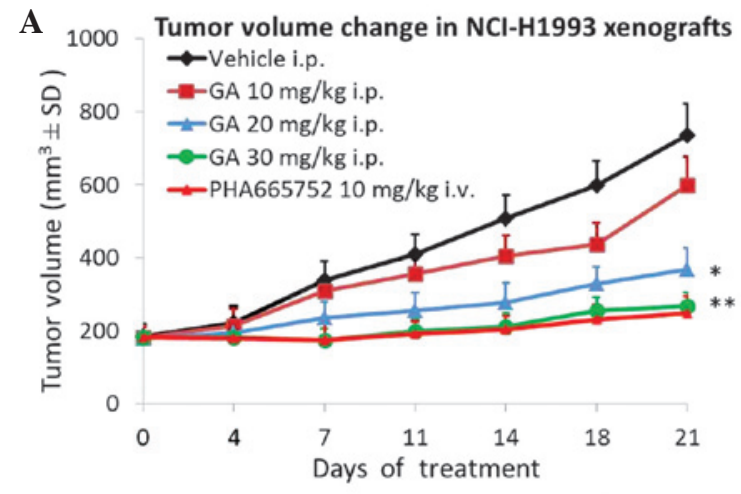

B
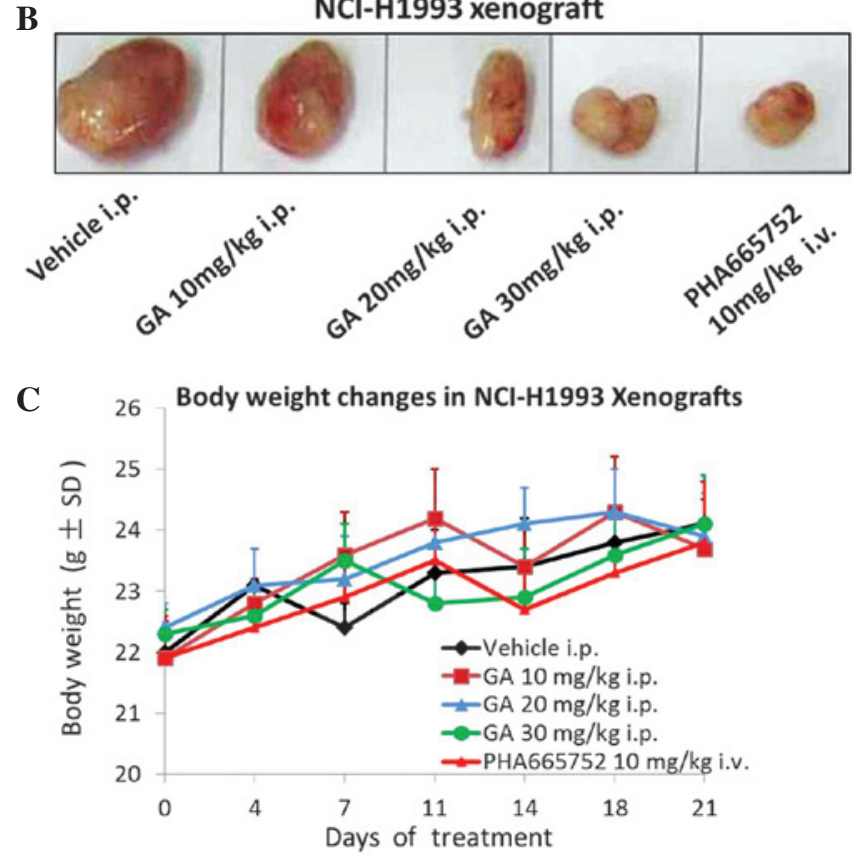

Figure 1. GA inhibits tumor growth in an NCI-H1993 xenograft model. (A) Nude mice bearing NCI-H1993 tumors received once daily i.p. injections with GA at the indicated dose, vehicle or positive control for up to 3 weeks. Tumor volume was measured using calipers on the indicated days. (B) NCI-H1993 tumors resected from nude mice on day 21 of the efficacy study. (C) Body weight changes during the efficacy study. Each dose of GA demonstrated no toxicity to the NCI-H1993 xenograft model. Values are expressed as the mean $\pm \mathrm{SD}, \mathrm{n}=10$. ${ }^{*} \mathrm{P}<0.05$ vs. vehicle group; ${ }^{* *} \mathrm{P}<0.01$ vs vehicle group. GA, gambogic acid; i.p., intraperitoneal; i.v., intravenous; $\mathrm{SD}$, standard deviation.

mice received i.p. injection with 10,20 or $30 \mathrm{mg} / \mathrm{kg}$ GA once a day for 21 days. As demonstrated in Fig. 1A and B, treatment with $10 \mathrm{mg} / \mathrm{kg} \mathrm{GA}$ only slightly inhibited tumor growth, however, $20 \mathrm{mg} / \mathrm{kg}$ GA markedly inhibited tumor growth $(\mathrm{P}=0.021)$ and $30 \mathrm{mg} / \mathrm{kg} \mathrm{GA}$ treatment almost completely inhibited tumor growth $(\mathrm{P}=0.008)$ compared with the vehicle control group. Throughout the duration of the efficacy study, no body weight loss was observed in any of the groups (Fig. 1C). The MET selective inhibitor PHA665752 was used as a positive control.

GA suppresses the protein expression of $p$-MET, $p$-AKT and p-ERK in NCI-H1993 xenografts. To ascertain whether the expression of p-MET in NCI-H1993 tumors was affected by GA treatment, the tumor tissues were analyzed by western blotting. The results demonstrated that the protein expression

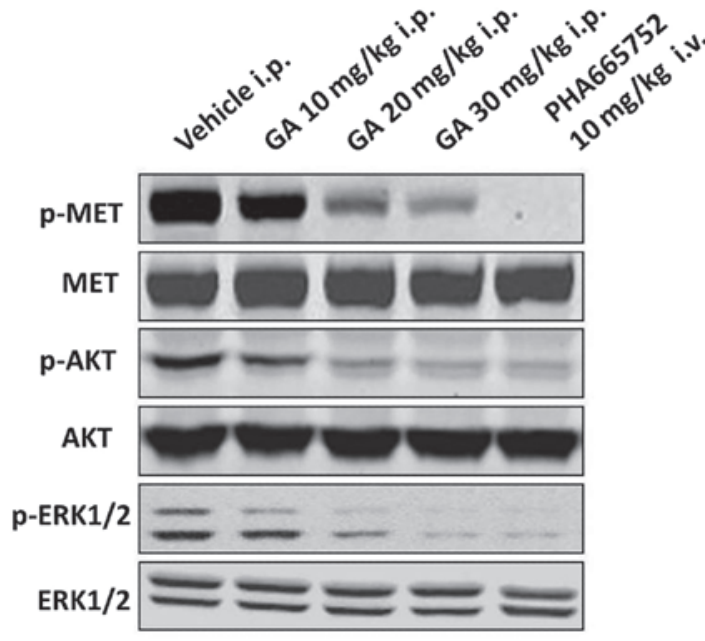

Figure 2. GA inhibits protein expression levels of p-MET, p-AKT and p-ERK in the NCI-H1993 xenograft model. NCI-H1993 xenografts were treated with GA at the indicated concentrations for $2 \mathrm{~h}$ on day 21 of the efficacy study, and subsequently the tumor tissues were subjected to western blot analysis. GA, gambogic acid; i.p., intraperitoneal; i.v., intravenous; p-, phosphorylated; ERK, extracellular-signal-regulated kinase.

levels of p-MET in the NCI-H1993 tumor tissues were downregulated by GA in a concentration-dependent manner compared with those of the vehicle group. In addition, the protein expression levels of the downstream signaling molecules p-AKT and p-ERK were also markedly downregulated in a concentration-dependent manner, compared with those of the vehicle group (Fig. 2). The MET selective inhibitor PHA665752 was used as a positive control.

GA treatment significantly inhibits the expression of Ki-67 in the NCI-H1993 xenograft model. GA was also assessed for its effect on the tumor mitotic index (Ki-67) using IHC. A significant 2-3-fold reduction in $\mathrm{Ki}-67$ levels was observed $2 \mathrm{~h}$ following the administration of 20 or $30 \mathrm{mg} / \mathrm{kg}$ GA in the NCI-H1993 tumor tissues ( $\mathrm{P}=0.046$ and 0.009 , respectively; Fig. $3 \mathrm{~A}$ and $\mathrm{B})$, which was consistent with the results of the efficacy study. The MET selective inhibitor PHA665752 also significantly reduced the expression of Ki-67 in tumor tissues $(\mathrm{P}=0.007)$.

GA does not alter caspase-3, -8 or -9 activities in NCI-H1993 xenografts. In order to investigate whether GA induces apoptosis in the NCI-H1993 xenograft model, the activities of caspase- $3,-8$ and -9 were measured using a colorimetric assay. The results demonstrated that GA had no effect on caspase-3, -8 and -9 activities in the NCI-H1993 xenograft model (Fig. 4).

\section{Discussion}

Previous studies have demonstrated that GA exerts significant anti-proliferative and pro-apoptotic effects on a variety of human cancer cell lines in vitro and in vivo (13-15). In addition, GA has been approved for use in clinical trials in China, although its anticancer mechanisms are not yet fully understood (16). To the best of our knowledge, the present study is the first to indicate that GA inhibits the tumor growth of 
A

$\mathrm{NCl}-\mathrm{H} 1993$ xenografts
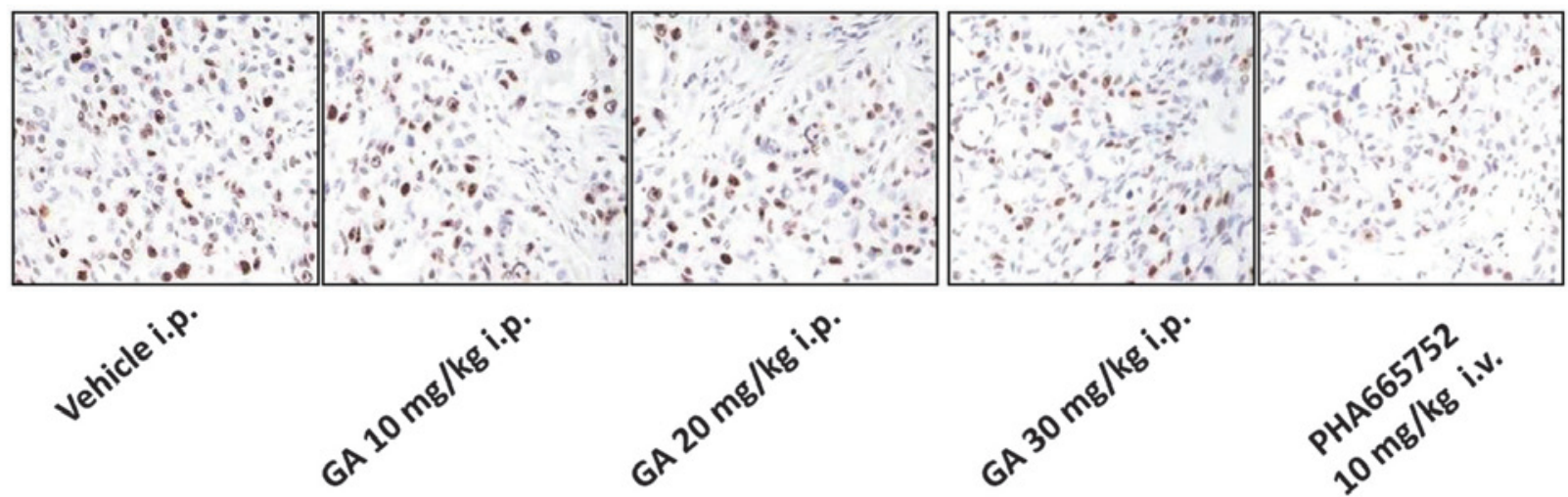

B

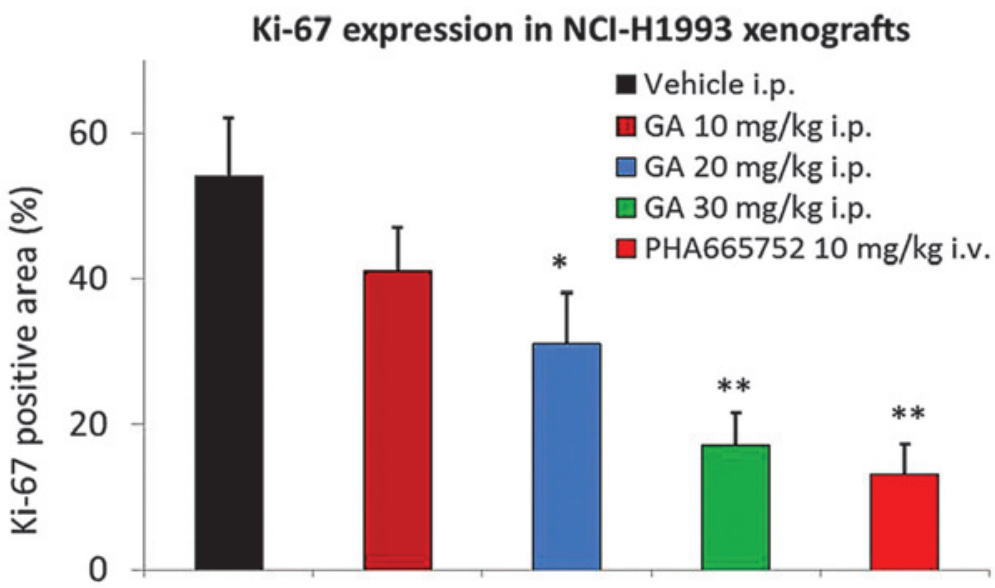

Figure 3. GA inhibits the expression of Ki-67 in the NCI-H1993 xenograft model. The NCI-H1993 xenograft model was treated with various doses of GA, vehicle or positive control for $2 \mathrm{~h}$ on day 21 of the efficacy study. (A) Representative images of Ki-67 staining. (B) Quantification of Ki-67 positive area (\%). Values are expressed as the mean \pm standard deviation, $\mathrm{n}=10 .{ }^{*} \mathrm{P}<0.05$ vs. vehicle group; ${ }^{* *} \mathrm{P}<0.01$ vs. vehicle group. GA, gambogic acid; i.p., intraperitoneal; i.v., intravenous.

NSCLC cells harboring a MET amplification in a dose-dependent manner. Wang et al (11) reported that GA synergistically potentiates cisplatin-induced apoptosis in NSCLC through suppressing NF- $\mathrm{KB}$ and MAPK/HO-1 signaling. Zhu et al (10) also reported that mechanisms of GA-induced apoptosis exist in NSCLC cells and are associated with transferrin receptors. However, in the present study, the antitumor mechanisms of GA on NCI-H1993 xenograft were demonstrated to be associated with MET downregulation. These results indicate that GA may be investigated further as a potential anticancer candidate for clinical applications.

A previous study demonstrated that MET and its receptor are overexpressed by $\sim 70 \%$ and $\sim 40 \%$ in human lung cancer tissues, respectively; such values are increased compared with those in breast (16\%) and ovarian cancer (31\%), but reduced compared with renal (72\%) and colorectal cancers (78\%) (17). However, p-MET expression is observed to be at the highest levels in lung cancer (73\%), followed by ovarian (33\%), breast (23\%) and renal (18\%) cancer (18). Therefore, MET may be a promising target for the treatment of lung cancer. In the present study, the expression of p-MET in NCI-H1993 tumors was inhibited by GA treatment in a dose-dependent manner. In addition, the downstream signaling molecules p-AKT and

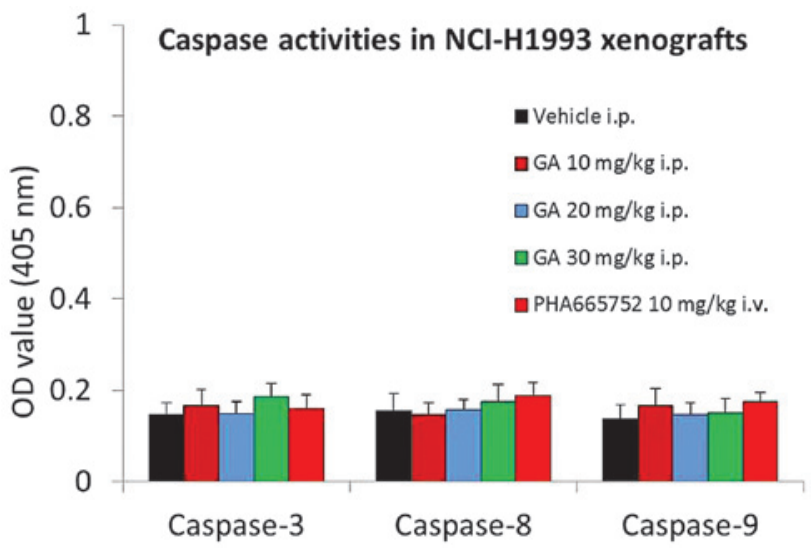

Figure 4. GA does not influence the activities of caspase- $3,-8$ or -9 in the NCI-H1993 xenograft model. NCI-H1993 xenografts were treated with the indicated dose of GA, vehicle or positive control for $2 \mathrm{~h}$ on day 21 of the efficacy study, prior to caspase colorimetric protease kit analysis. Values are expressed as the mean \pm standard deviation, $n=10$. GA, gambogic acid; i.p., intraperitoneal; i.v., intravenous; OD, optical density.

p-ERK were also downregulated by GA, thereby resulting in tumor growth inhibition. However, AKT and ERK are downstream signaling molecules not only for MET, but also for 
other members of the receptor tyrosine kinase (RTK) family. It is not clear whether p-MET is the only RTK family member that is inhibited by GA treatment and therefore further investigation is required.

In a number of previous reports, GA was found to induce apoptosis in tumor cells $(19,20)$. However, in the present study, no effect of GA on caspase-3, 8 and 9 activities was identified in the NCI-H1993 xenograft model. Another previous study also reported that GA significantly inhibited U87 tumor growth without inducing apoptosis (21).

In conclusion, the present study demonstrated that GA exerted significant antitumor effects on NCI-H1993 xenografts by downregulating the p-MET protein expression level. The expression of the downstream molecules p-AKT and p-ERK were also inhibited by GA. Ki-67 analysis in tumor tissues demonstrated that the antitumor effect of GA was primarily associated with its anti-proliferation activity. These results may aid in explaining the molecular mechanisms underlying the antiumor effects of GA on NSCLC.

\section{References}

1. Maestrini E, Tamagnone L, Longati P, et al: A family of transmembrane proteins with homology to the MET-hepatocyte growth factor receptor. Proc Natl Acad Sci USA 93: 674-678, 1996.

2. Sattler M and Salgia R: c-Met and hepatocyte growth factor: Potential as novel targets in cancer therapy. Curr Oncol Rep 9: 102-108, 2007

3. Guo Q, Qi Q, You Q, Gu H, Zhao L and Wu Z: Toxicological studies of gambogic acid and its potential targets in experimental animals. Basic Clin Pharmacol Toxicol 99: 178-184, 2006.

4. Wu ZQ, Guo QL, You QD, et al: Gambogic acid inhibits proliferation of human lung carcinoma SPC-A1 cells in vivo and in vitro and represses telomerase activity and telomerase reverse transcriptase mRNA expression in the cells. Biol Pharm Bull 27: 1769-1774, 2004

5. Guo QL, Lin SS, You QD, et al: Inhibition of human telomerase reverse transcriptase gene expression by gambogic acid in human hepatoma SMMC-7721 cells. Life Sci 78: 1238-1245, 2006.

6. Wang X, Lu N, Yang Q, et al: Studies on chemical modification and biology of a natural product, gambogic acid (III): Determination of the essential pharmacophore for biological activity. Eur J Med Chem 46: 1280-1290, 2011.
7. Wang J, Zhao L, Hu Y, et al: Studies on chemical structure modification and biology of a natural product, Gambogic acid (I): Synthesis and biological evaluation of oxidized analogues of gambogic acid. Eur J Med Chem 44: 2611-2620, 2009.

8. Pandey MK, Sung B, Ahn KS, et al: Gambogic acid, a novel ligand for transferrin receptor, potentiates TNF induced apoptosis through modulation of the nuclear factor-kappaB signaling pathway. Blood 110: 3517-3525, 2007.

9. Yi T, Yi Z, Cho SG, et al: Gambogic acid inhibits angiogenesis and prostate tumor growth by suppressing vascular endothelial growth factor receptor 2 signaling. Cancer Res 68: 1843-1850, 2008

10. Zhu X, Zhang H, Lin Y, et al: Mechanisms of gambogic acid-induced apoptosis in non-small cell lung cancer cells in relation to transferrin receptors. J Chemother 21: 666-672, 2009.

11. Wang LH, Li Y, Yang SN, et al: Gambogic acid synergistically potentiates cisplatin-induced apoptosis in non-small-cell lung cancer through suppressing NF- $\mathrm{BB}$ and MAPK/HO-1 signaling. Br J Cancer 110: 341-352, 2014

12. Sunaga N, Shames DS, Girard L, et al: Knockdown of oncogenic KRAS in non-small cell lung cancers suppresses tumor growth and sensitizes tumor cells to targeted therapy. Mol Cancer Ther 10: 336-346, 2011.

13. Gu HY, Guo QL, You QD, et al: Gambogic acid inducing apoptosis in human hepatoma SMMC-7721 cells with p53 and Bax up-regulated. Chin J Nat Med 3: 169-171, 2005.

14. Liu W, Guo QL, You QD, et al: Anticancer effect and apoptosis induction of gambogic acid in human gastric cancer line BGC-823. World J Gastroenterol 11: 3655-3659, 2005.

15. Zhao L, Guo QL, You QD, et al: Gambogic acid induces apoptosis and regulates expressions of $\mathrm{Bax}$ and $\mathrm{Bcl}-2$ protein in human gastric carcinoma MGC-803 cells. Biol Pharm Bull 27: 998-1003, 2004.

16. Zhou Z and Wang J: Phase I human tolerability trial of gambogic acid. Chin J New Drugs 16: 79-82, 2007.

17. Paez JG, Jänne PA, Lee JC, et al: EGFR mutations in lung cancer: Correlation with clinical response to gefitinib therapy. Science 304: 1497-1500, 2004.

18. Maulik G, Kijima T, Ma PC, et al: Modulation of the c-Met/hepatocyte growth factor pathway in small cell lung cancer. Clin Cancer Res 8: 620-627, 2002

19. Zhao W, Zhou SF, Zhang ZP, et al: Gambogic acid inhibits the growth of osteosarcoma cells in vitro by inducing apoptosis and cell cycle arrest. Oncol Rep 25: 1289-1295, 2011.

20. Yang Y, Yang L, You QD, et al: Differential apoptotic induction of gambogic acid, a novel anticancer natural product, on hepatoma cells and normal hepatocytes. Cancer Lett 256: 259-266, 2007.

21. He XY, Liu XJ, Chen X, et al: Gambogic acid induces EGFR degradation and Akt/mTORC1 inhibition through AMPK dependent-LRIG1 upregulation in cultured U87 glioma cells. Biochem Biophys Res Commun 435: 397-402, 2013. 